\title{
A FIXED POINT APPROACH TO THE CAUCHY-RASSIAS STABILITY OF GENERAL JENSEN TYPE QUADRATIC-QUADRATIC MAPPINGS
}

\author{
Choonkil Park, M. Eshaghi Gordji, and H. Khodaei
}

\begin{abstract}
In this paper, we investigate the Cauchy-Rassias stability in Banach spaces and also the Cauchy-Rassias stability using the alternative fixed point for the functional equation:

$$
f\left(\frac{s x+t y}{2}+r z\right)+f\left(\frac{s x+t y}{2}-r z\right)+f\left(\frac{s x-t y}{2}+r z\right)+f\left(\frac{s x-t y}{2}-r z\right)
$$$$
=s^{2} f(x)+t^{2} f(y)+4 r^{2} f(z)
$$

for any fixed nonzero integers $s, t, r$ with $r \neq \pm 1$.

\section{Introduction}

Ulam [29] raised the following problem concerning the stability of homomorphisms: Give conditions in order for a linear mapping near an approximately linear mapping to exist? The following theorem which is called the CauchyRassias stability is a generalized solution to this problem.

Theorem 1.1. Let $E$ be a real normed space, $F$ be a real Banach space and $f: E \rightarrow F$ be a mapping such that for each fixed $x \in E$ the mapping $t \mapsto f(t x)$ is continuous on $\mathbb{R}$. Assume that there exist constants $\varepsilon \geq 0$ and $p \geq 0$ with $p \neq 1$ such that

$$
\|f(x+y)-f(x)-f(y)\| \leq \varepsilon\left(\|x\|^{p}+\|y\|^{p}\right) \quad(x, y \in E) .
$$

Then there exists a unique $\mathbb{R}$-linear mapping $T: E \rightarrow F$ satisfying

$$
\|f(x)-T(x)\| \leq \varepsilon\|x\|^{p} /\left(1-2^{p-1}\right) \quad(x \in E) .
$$

The above Cauchy-Rassias stability in real Banach spaces was obtained by Hyers [10] for the case $p=0$, by Rassias [24] for the case $p \in(0,1)$, and by Gajda [9] for the case $p>1$. In particular, Rassias and Šmrl [25] gave an

Received March 24, 2009.

2000 Mathematics Subject Classification. 39B82, 39B52, 47H10.

Key words and phrases. Cauchy-Rassias stability, quadratic mapping, fixed point method.

The first author was supported by Basic Science Research Program through the National Research Foundation of Korea funded by the Ministry of Education, Science and Technology (NRF-2009-0070788). 
example to show that it does not occur for the case $p=1$. Also, Trif [28] studied the Cauchy-Rassias stability of the Jensen type functional equation. In addition, Park [17] studied the Cauchy-Rassias stability of modified Trif functional equations associated with homomorphisms in Banach module over $\mathrm{C}^{*}$-algebras.

The functional equation

$$
f(x+y)+f(x-y)=2 f(x)+2 f(y)
$$

is related to symmetric bi-additive function and is called a quadratic functional equation and every solution of the quadratic equation (1.1) is said to be a quadratic function particulary. It is well known that a function $f$ between two real vector spaces is quadratic if and only if there exists a unique symmetric bi-additive function $B$ such that $f(x)=B(x, x)$, where

$$
B(x, y)=\frac{1}{4}(f(x+y)-f(x-y))
$$

for all $x$ (see $[1,13])$. Skof proved Hyers-Ulam stability problem for the quadratic functional equation (1.1) for a class of functions $f: A \rightarrow B$, where $\mathrm{A}$ is normed space and B is a Banach space (see [27]). In 1992, Czerwik [7] proved the Cauchy-Rassias stability of the equation (1.1) (see also [18, 19]). Recently, Park, Hong, and Kim [19] have investigated the Cauchy-Rassias stability of the Jensen type quadratic-quadratic equation:

$f\left(\frac{x+y}{2}+z\right)+f\left(\frac{x+y}{2}-z\right)+f\left(\frac{x-y}{2}+z\right)+f\left(\frac{x-y}{2}-z\right)=f(x)+f(y)+4 f(z)$

in Banach spaces. Several functional equations have been investigated in $[2,3$, $11,14,16,22,23]$.

We recall some basic facts concerning Jensen type quadratic-quadratic mapping.

Definition 1.2. An even mapping $Q: X \rightarrow Y$ is called quadratic-quadratic if $Q$ satisfies $Q(0)=0$ and the functional equation (1.1). We note that (1.1) is equivalent to the Jensen quadratic equation

$$
2 f\left(\frac{z+w}{2}\right)+2 f\left(\frac{z-w}{2}\right)=f(z)+f(w)
$$

for $z=x+y, w=x-y$. An even mapping $Q: X \rightarrow Y$ is called Jensen type quadratic-quadratic mapping if $Q$ satisfies $Q(0)=0$ and the functional equation (1.2).

Now we introduce the general Jensen type quadratic-quadratic functional equation:

$$
\begin{aligned}
& f\left(\frac{s x+t y}{2}+r z\right)+f\left(\frac{s x+t y}{2}-r z\right)+f\left(\frac{s x-t y}{2}+r z\right)+f\left(\frac{s x-t y}{2}-r z\right) \\
= & s^{2} f(x)+t^{2} f(y)+4 r^{2} f(z)
\end{aligned}
$$


for any fixed nonzero integers $s, t, r$ with $r \neq \pm 1$. Afterward, we investigate the Cauchy-Rassias stability in Banach spaces and also the Cauchy-Rassias stability using the alternative fixed point.

\section{General Jensen type quadratic-quadratic functional equation}

Let $X$ and $Y$ be real vector spaces. We here present the general solution of (1.3).

Theorem 2.1. If an even mapping $f: X \rightarrow Y$ satisfies $f(0)=0$ and the functional equation (1.3), then the mapping $f$ satisfies (1.2). Therefore, any even mapping $f$ satisfies (1.3) and $f(0)=0$ is a Jensen type quadratic-quadratic mapping.

Proof. Letting $y=z=0$ in (1.3) and using $f(0)=0$, we get

$$
f\left(\frac{s}{2} x\right)=\frac{s^{2}}{4} f(x)
$$

for all $x \in X$. Setting $x=y=0$ in (1.3) and using the evenness of $f$, we obtain

$$
f(r z)=r^{2} f(z)
$$

for all $z \in X$. So

$$
f\left(\frac{s r}{2} x\right)=\frac{s^{2} r^{2}}{4} f(x)
$$

for all $x \in X$. Replacing $x, y$ and $z$ by $r x, 0$ and $\frac{s}{2} z$ in (1.3), respectively, we have

$$
f\left(\frac{s r}{2} x+\frac{s r}{2} z\right)+f\left(\frac{s r}{2} x-\frac{s r}{2} z\right)=s^{2} f(r x)+4 r^{2} f\left(\frac{s}{2} z\right)
$$

for all $x, z \in X$. But since $s, r \neq 0$, it follows from (2.1), (2.2), (2.3) and (2.4) that

$$
f(x+z)+f(x-z)=2 f(x)+2 f(z)
$$

for all $x, z \in X$. Now, we substitute $x=\frac{x+y}{2}$ and $z=\frac{x-y}{2}$ in (2.5), we lead to

$$
2 f\left(\frac{x+y}{2}\right)+2 f\left(\frac{x-y}{2}\right)=f(x)+f(y)
$$

for all $x, y \in X$.

Therefore $f$ satisfies (1.2).

\section{Cauchy-Rassias stability in Banach spaces}

From this point on, let $X$ be a real vector space and let $Y$ be a Banach space. Before taking up the main subject, for the given mapping $f: X \rightarrow Y$ we define the difference operator $\Delta_{f}: X \times X \times X \rightarrow Y$ by

$$
\begin{aligned}
\Delta_{f}(x, y, z):= & f\left(\frac{s x+t y}{2}+r z\right)+f\left(\frac{s x+t y}{2}-r z\right)+f\left(\frac{s x-t y}{2}+r z\right) \\
& +f\left(\frac{s x-t y}{2}-r z\right)-s^{2} f(x)-t^{2} f(y)-4 r^{2} f(z)
\end{aligned}
$$


for all $x, y, z \in X$ and any fixed nonzero integers $s, t, r$ with $r \neq \pm 1$.

Theorem 3.1. Let $j \in\{-1,1\}$ be fixed, and let $\varphi: X \times X \times X \rightarrow[0, \infty)$ be a function such that

$$
\begin{gathered}
\widetilde{\varphi}(z):=\sum_{i=\frac{1-j}{2}}^{\infty} \frac{1}{r^{2 i j}} \varphi\left(0,0, r^{i j} z\right)<\infty \\
\lim _{n \rightarrow \infty} \frac{1}{r^{2 n j}} \varphi\left(r^{n j} x, r^{n j} y, r^{n j} z\right)=0
\end{gathered}
$$

for all $x, y, z \in X$. Suppose that $f: X \rightarrow Y$ be an even mapping with $f(0)=0$ satisfies

$$
\left\|\Delta_{f}(x, y, z)\right\| \leq \varphi(x, y, z)
$$

for all $x, y, z \in X$. Then there exists a unique Jensen type quadratic-quadratic mapping $Q: X \rightarrow Y$ such that

$$
\|f(z)-Q(z)\| \leq \frac{1}{4 r^{1+j}} \widetilde{\varphi}\left(\frac{z}{r^{\frac{1-j}{2}}}\right)
$$

for all $z \in X$.

Proof. For $j=1$. Setting $x=y=0$ in (3.3) and using the evenness of $f$, we obtain

$$
\left\|4 f(r z)-4 r^{2} f(z)\right\| \leq \varphi(0,0, z)
$$

for all $z \in X$. So

$$
\left\|f(z)-\frac{1}{r^{2}} f(r z)\right\| \leq \frac{1}{4 r^{2}} \varphi(0,0, z)
$$

for all $z \in X$. Replacing $z$ by $r z$ in (3.6) and dividing by $r^{2}$ and summing the resulting inequality with $(3.6)$, we get

$$
\left\|f(z)-\frac{1}{r^{4}} f\left(r^{2} z\right)\right\| \leq \frac{1}{4 r^{2}}\left(\varphi(0,0, z)+\frac{\varphi(0,0, r z)}{r^{2}}\right)
$$

for all $z \in X$. Hence

$$
\left\|\frac{1}{r^{2 k}} f\left(r^{k} z\right)-\frac{1}{r^{2 m}} f\left(r^{m} z\right)\right\| \leq \frac{1}{4 r^{2}} \sum_{i=k}^{m-1} \frac{1}{r^{2 i}} \varphi\left(0,0, r^{i} z\right)
$$

for all nonnegative integers $m$ and $k$ with $m>k$ and for all $z \in X$. It follows from (3.1) and (3.8) that the sequence $\left\{\frac{1}{r^{2 n}} f\left(r^{n} z\right)\right\}$ is a Cauchy sequence for all $z \in X$. Since $Y$ is complete, the sequence $\left\{\frac{1}{r^{2 n}} f\left(r^{n} z\right)\right\}$ converges. Therefore, one can define the mapping $Q: X \rightarrow Y$ by

$$
Q(z):=\lim _{n \rightarrow \infty} \frac{1}{r^{2 n}} f\left(r^{n} z\right)
$$


for all $z \in X$. By (3.2) for $j=1$ and (3.3),

$$
\begin{aligned}
\left\|\Delta_{Q}(x, y, z)\right\| & =\lim _{n \rightarrow \infty} \frac{1}{r^{2 n}}\left\|\Delta_{f}\left(r^{n} x, r^{n} y, r^{n} z\right)\right\| \\
& \leq \lim _{n \rightarrow \infty} \frac{1}{r^{2 n}} \varphi\left(r^{n} x, r^{n} y, r^{n} z\right)=0
\end{aligned}
$$

for all $x, y, z \in X$. So $\Delta_{Q}(x, y, z)=0$. By Theorem 2.1, the mapping $Q: X \rightarrow$ $Y$ is a Jensen type quadratic-quadratic mapping. Moreover, letting $k=0$ and passing the limit $m \rightarrow \infty$ in (3.8), we get the inequality (3.4) for $j=1$.

Now, let $Q^{\prime}: X \rightarrow Y$ be another Jensen type quadratic-quadratic mapping satisfying (1.3) and (3.4). So

$$
\begin{aligned}
\left\|Q(z)-Q^{\prime}(z)\right\| & =\frac{1}{r^{2 n}}\left\|Q\left(r^{n} z\right)-Q^{\prime}\left(r^{n} z\right)\right\| \\
& \leq \frac{1}{r^{2 n}}\left(\left\|Q\left(r^{n} z\right)-f\left(r^{n} z\right)\right\|+\left\|Q^{\prime}\left(r^{n} z\right)-f\left(r^{n} z\right)\right\|\right) \\
& \leq \frac{1}{2 r^{2} r^{2 n}} \widetilde{\varphi}\left(r^{n} z\right)
\end{aligned}
$$

which tends to zero as $n \rightarrow \infty$ for all $z \in X$. So we can conclude that $Q(z)=$ $Q^{\prime}(z)$ for all $z \in X$. This proves the uniqueness of $Q$.

Also, for $j=-1$, it follows from (3.5) that

$$
\left\|f(z)-r^{2} f\left(\frac{z}{r}\right)\right\| \leq \frac{1}{4} \varphi\left(0,0, \frac{z}{r}\right)
$$

for all $z \in X$. Hence

$$
\left\|r^{2 k} f\left(\frac{z}{r^{k}}\right)-r^{2 m} f\left(\frac{z}{r^{m}}\right)\right\| \leq \frac{1}{4} \sum_{i=k}^{m-1} r^{2 i} \varphi\left(0,0, \frac{z}{r^{i+1}}\right)
$$

for all nonnegative integers $m$ and $k$ with $m>k$ and for all $z \in X$. It follows from (3.10) that the sequence $\left\{r^{2 n} f\left(\frac{z}{r^{n}}\right)\right\}$ is a Cauchy sequence for all $z \in X$. Since $Y$ is complete, the sequence $\left\{r^{2 n} f\left(\frac{z}{r^{n}}\right)\right\}$ converges. So one can define the mapping $Q: X \rightarrow Y$ by

$$
Q(z):=\lim _{n \rightarrow \infty} r^{2 n} f\left(\frac{z}{r^{n}}\right)
$$

for all $z \in X$. By (3.2) for $j=-1$ and (3.3),

$$
\left\|\Delta_{Q}(x, y, z)\right\|=\lim _{n \rightarrow \infty} r^{2 n}\left\|\Delta_{f}\left(\frac{x}{r^{n}}, \frac{y}{r^{n}}, \frac{z}{r^{n}}\right)\right\| \leq \lim _{n \rightarrow \infty} r^{2 n} \varphi\left(\frac{x}{r^{n}}, \frac{y}{r^{n}}, \frac{z}{r^{n}}\right)=0
$$

for all $x, y, z \in X$. So $\Delta_{Q}(x, y, z)=0$. By Theorem 2.1, the mapping $Q: X \rightarrow$ $Y$ is a Jensen type quadratic-quadratic mapping. Moreover, letting $k=0$ and passing the limit $m \rightarrow \infty$ in (3.10), we get the inequality (3.4) for $j=-1$.

The rest of the proof is similar to the proof of the previous section. 


\section{Cauchy-Rassias stability using alternative fixed point}

Recently, Cădariu and Radu [4] applied the fixed point method to the investigation of the Cauchy additive functional equation. Using such a clever idea, they could present another proof for the Hyers-Ulam stability of that equation $[5,6]$. In this section, by using the idea of Cădariu and Radu, we will prove the Cauchy-Rassias stability of the general Jensen type quadratic-quadratic functional equation (1.3) (see also $[12,15,21]$ ).

Theorem 4.1 (The alternative of fixed point $[8,26]$ ). Suppose that we are given a complete generalized metric space $(\Omega, d)$ and a strictly contractive mapping $T: \Omega \rightarrow \Omega$ with Lipschitz constant $L$. Then for each given $x \in \Omega$, either $d\left(T^{n} x, T^{n+1} x\right)=\infty$ for all $n \geq 0$, or other exists a natural number $n_{0}$ such that

- $d\left(T^{n} x, T^{n+1} x\right)<\infty$ for all $n \geq n_{0}$

- the sequence $\left\{T^{n} x\right\}$ is convergent to a fixed point $y^{*}$ of $T$;

- $y^{*}$ is the unique fixed point of $T$ in the set $\Lambda=\left\{y \in \Omega: d\left(T^{n_{0}} x, y\right)<\infty\right\}$;

- $d\left(y, y^{*}\right) \leq \frac{1}{1-L} d(y, T y)$ for all $y \in \Lambda$.

Utilizing the above mentioned fixed point alternative, we now obtain our main result, i.e., the Cauchy-Rassias stability of the functional equation (1.3).

Theorem 4.2. Suppose that $j \in\{-1,1\}$ be fixed, and let $f: X \rightarrow Y$ an even function with $f(0)=0$ for which there exists a function $\varphi: X \times X \times X \rightarrow[0, \infty)$ such that

$$
\begin{gathered}
\lim _{n \rightarrow \infty} \frac{1}{r^{2 n j}} \varphi\left(r^{n j} x, r^{n j} y, r^{n j} z\right)=0, \\
\left\|\Delta_{f}(x, y, z)\right\| \leq \varphi(x, y, z)
\end{gathered}
$$

for all $x, y, z \in X$. If there exists $L<1$ such that the function $\varphi$ has the property

$$
\varphi\left(0,0, \frac{z}{r}\right) \leq L r^{2} \varphi\left(0,0, \frac{z}{r^{2}}\right)
$$

for all $z \in X$, then there exists a unique Jensen type quadratic-quadratic function $Q: X \rightarrow Y$ such that, we have the inequality

$$
\|f(z)-Q(z)\| \leq \frac{L^{\frac{j+1}{2}}}{4(1-L)} \varphi\left(0,0, \frac{z}{r}\right)
$$

for all $z \in X$.

Proof. Consider the set $\Omega=\{g \mid g: X \rightarrow Y, g(0)=0\}$, and introduce the generalized metric

$$
d(g, h)=d_{\varphi}(g, h)=\inf \left\{K \in(0, \infty):\|g(z)-h(z)\| \leq K \varphi\left(0,0, \frac{z}{r}\right), z \in X\right\}
$$

on $\Omega$. It is easy to see that $(\Omega, d)$ is complete. 
Now we define a function $T: \Omega \rightarrow \Omega$ by $T g(z)=\frac{1}{r^{2 j}} g\left(r^{j} z\right)$ for all $z \in X$. Note that for all $g, h \in \Omega$,

$$
\begin{aligned}
d(g, h)<K & \Rightarrow\|g(z)-h(z)\| \leq K \varphi\left(0,0, \frac{z}{r}\right) & \text { for all } z \in X \\
& \Rightarrow\left\|\frac{1}{r^{2 j}} g\left(r^{j} z\right)-\frac{1}{r^{2 j}} h\left(r^{j} z\right)\right\| \leq \frac{1}{r^{2 j}} K \varphi\left(0,0, r^{j-1} z\right) & \text { for all } z \in X \\
& \Rightarrow\left\|\frac{1}{r^{2 j}} g\left(r^{j} z\right)-\frac{1}{r^{2 j}} h\left(r^{j} z\right)\right\| \leq L K \varphi\left(0,0, \frac{z}{r}\right) & \text { for all } z \in X \\
& \Rightarrow d(T g, T h) \leq L K . &
\end{aligned}
$$

Hence we see that

$$
d(T g, T h) \leq L d(g, h)
$$

for all $g, h \in \Omega$, that is, $T$ is a strictly self-mapping of $\Omega$ with the Lipschitz constant $L$. Putting $x=y=0$ in (3.3) and using the evenness of $f$, we get

$$
\left\|4 f(r z)-4 r^{2} f(z)\right\| \leq \varphi(0,0, z)
$$

for all $z \in X$. Now, by using (4.3) for $z:=r z$, we obtain that

$$
\left\|f(z)-\frac{1}{r^{2}} f(r z)\right\| \leq \frac{1}{4 r^{2}} \varphi(0,0, z) \leq \frac{L}{4} \varphi\left(0,0, \frac{z}{r}\right)
$$

for all $z \in X$, that is, $d(f, T f) \leq \frac{L}{4}<\infty$.

If we substitute $z:=\frac{z}{r}$ in (4.5), we see that

$$
\left\|f(z)-r^{2} f\left(\frac{z}{r}\right)\right\| \leq \frac{1}{4} \varphi\left(0,0, \frac{z}{r}\right)
$$

for all $z \in X$, that is, $d(f, T f) \leq \frac{1}{4}<\infty$.

Now, from the fixed point alternative in both cases, it follows that there exists a fixed point $Q$ of $T$ in $\Omega$ such that

$$
Q(z)=\lim _{n \rightarrow \infty} \frac{1}{r^{2 n j}} f\left(r^{n j} z\right)
$$

for all $z \in X$, since $\lim _{n \rightarrow \infty} d\left(T^{n} f, Q\right)=0$.

Also, if we replace $x, y$ and $z$ by $r^{n j} x, r^{n j} y$ and $r^{n j} z$ in (2.30), respectively, and divide by $r^{2 n j}$. Then it follows from (4.1) and (4.6) that

$$
\begin{aligned}
\left\|\Delta_{Q}(x, y, z)\right\| & =\lim _{n \rightarrow \infty} \frac{1}{r^{2 n j}}\left\|\Delta_{f}\left(r^{n j} x, r^{n j} y, r^{n j} z\right)\right\| \\
& \leq \lim _{n \rightarrow \infty} \frac{1}{r^{2 n j}} \varphi\left(r^{n j} x, r^{n j} y, r^{n j} z\right)=0
\end{aligned}
$$

for all $x, y, z \in X$, so $\Delta_{Q}(x, y, z)=0$. By Theorem 2.1, the function $Q$ is a Jensen type quadratic-quadratic function.

According to the fixed point alterative, since $Q$ is the unique fixed point of $T$ in the set $\Lambda=\{g \in \Omega: d(f, g)<\infty\}, Q$ is the unique function such that

$$
\|f(z)-Q(z)\| \leq K \varphi\left(0,0, \frac{z}{r}\right)
$$


for all $z \in X$ and $K>0$. Again using the fixed point alterative, gives

$$
d(f, Q) \leq \frac{1}{1-L} d(f, T f) \leq \frac{L^{\frac{j+1}{2}}}{4(1-L)}
$$

so we conclude that

$$
\|f(z)-Q(z)\| \leq \frac{L^{\frac{j+1}{2}}}{4(1-L)} \varphi\left(0,0, \frac{z}{r}\right)
$$

for all $z \in X$. This completes the proof.

Corollary 4.3. Let $\varepsilon, p_{1}, p_{2}, p_{3} \geq 0$ be real numbers such that $p_{1}, p_{2}, p_{3}<2$ or $p_{1}, p_{2}, p_{3}>2$. Suppose that an even function $f: X \rightarrow Y$ with $f(0)=0$ satisfies

$$
\left\|\Delta_{f}(x, y, z)\right\| \leq \varepsilon\left(\|x\|^{p_{1}}+\|y\|^{p_{2}}+\|z\|^{p_{3}}\right)
$$

for all $x, y, z \in X$. Then there exists a unique Jensen type quadratic-quadratic function $Q: X \rightarrow Y$ such that

$$
\|f(z)-Q(z)\| \leq \frac{\varepsilon}{4\left|r^{2}-r^{p}\right|}\|z\|^{p_{3}}
$$

for all $z \in X$.

Proof. In Theorem 4.2, put $\varphi(x, y, z):=\varepsilon\left(\|x\|^{p_{1}}+\|y\|^{p_{2}}+\|z\|^{p_{3}}\right)$ for all $x, y, z \in X$. Then the relation (4.1) is true for $p_{1}, p_{2}, p_{3}<2$ or $p_{1}, p_{2}, p_{3}>2$ and also the inequality (4.3) holds with $L=r^{\left(p_{3}-2\right) j}$. So from (4.4), we get (4.7).

Corollary 4.4. Assume that $\theta \geq 0$ be fixed. Let $f: X \rightarrow Y$ be an even function such that

$$
\left\|\Delta_{f}(x, y, z)\right\| \leq \theta
$$

for all $x, y, z \in X$. Then there exists a unique Jensen type quadratic-quadratic function $Q: X \rightarrow Y$ such that

$$
\|f(z)-Q(z)\| \leq \frac{\theta}{12\left(r^{2}-1\right)}
$$

holds for all $z \in X$.

Proof. Letting $p_{3}=0$ and $\varepsilon=\frac{\theta}{3}$ and applying Corollary 4.3, we get the result.

\section{References}

[1] J. Aczél and J. Dhombres, Functional Equations in Several Variables, Cambridge University Press, Cambridge, 1989.

[2] J. Bae and W. Park, Generalized Jensen's functional equations and approximate algebra homomorphisms, Bull. Korean Math. Soc. 39 (2002), no. 3, 401-410.

[3] D. Boo, S. Oh, C. Park, and J. Park, Generalized Jensen's equations in Banach modules over a $C^{*}$-algebra and its unitary group, Taiwanese J. Math. 7 (2003), no. 4, 641-655.

[4] L. Cădariu and V. Radu, On the stability of the Cauchy functional equation: a fixed point approach, Iteration theory (ECIT '02), 43-52, Grazer Math. Ber., 346, Karl-FranzensUniv. Graz, Graz, 2004 
[5] - The fixed points method for the stability of some functional equations, Carpathian J. Math. 23 (2007), no. 1-2, 63-72.

[6] L. Cădariu and V. Radu, Fixed points and the stability of quadratic functional equations, An. Univ. Timişoara Ser. Mat.-Inform. 41 (2003), no. 1, 25-48.

[7] St. Czerwik, On the stability of the quadratic mapping in normed spaces, Abh. Math. Sem. Univ. Hamburg 62 (1992), 59-64.

[8] J. B. Diaz and B. Margolis, A fixed point theorem of the alternative, for contractions on a generalized complete metric space, Bull. Amer. Math. Soc. 74 (1968), 305-309.

[9] Z. Gajda, On stability of additive mappings, Internat. J. Math. Math. Sci. 14 (1991), no. $3,431-434$.

[10] D. H. Hyers, On the stability of the linear functional equation, Proc. Natl. Acad. Sci. U.S.A. 27 (1941), 222-224.

[11] S. Jung, Hyers-Ulam-Rassias stability of Jensen's equation and its application, Proc. Amer. Math. Soc. 126 (1998), no. 11, 3137-3143.

[12] Y. Jung and I. Chang, The stability of a cubic type functional equation with the fixed point alternative, J. Math. Anal. Appl. 306 (2005), no. 2, 752-760.

[13] Pl. Kannappan, Quadratic functional equation and inner product spaces, Results Math. 27 (1995), no. 3-4, 368-372.

[14] Y. Lee and K. Jun, A generalization of the Hyers-Ulam-Rassias stability of Jensen's equation, J. Math. Anal. Appl. 238 (1999), no. 1, 305-315.

[15] M. Mirzavaziri and M. S. Moslehian, A fixed point approach to stability of a quadratic equation, Bull. Braz. Math. Soc. (N.S.) 37 (2006), no. 3, 361-376.

[16] M. S. Moslehian and L. Székelyhidi, Stability of ternary homomorphisms via generalized Jensen equation, Results Math. 49 (2006), no. 3-4, 289-300.

[17] C. Park, Modified Trif's functional equations in Banach modules over a $C^{*}$-algebra and approximate algebra homomorphisms, J. Math. Anal. Appl. 278 (2003), no. 1, 93-108.

[18] - Generalized quadratic mappings in several variables, Nonlinear Anal. 57 (2004), no. 5-6, 713-722.

[19] C. Park, S. Hong, and M. Kim, Jensen type quadratic-quadratic mapping in Banach spaces, Bull. Korean Math. Soc. 43 (2006), no. 4, 703-709.

[20] C. Park, J. Park, and J. Shin, Hyers-Ulam-Rassias stability of quadratic functional equations in Banach modules over a $C^{*}$-algebra, Chinese Ann. Math. Ser. B 24 (2003), no. 2, 261-266.

[21] V. Radu, The fixed point alternative and the stability of functional equations, Fixed Point Theory 4 (2003), no. 1, 91-96.

[22] J. M. Rassias and M. J. Rassias, Asymptotic behavior of Jensen and Jensen type functional equations, Panamer. Math. J. 15 (2005), no. 4, 21-35.

[23] _ Asymptotic behavior of alternative Jensen and Jensen type functional equations, Bull. Sci. Math. 129 (2005), no. 7, 545-558.

[24] Th. M. Rassias, On the stability of the linear mapping in Banach spaces, Proc. Amer. Math. Soc. 72 (1978), no. 2, 297-300.

[25] Th. M. Rassias and P. Šemrl, On the behavior of mappings which do not satisfy HyersUlam stability, Proc. Amer. Math. Soc. 114 (1992), no. 4, 989-993.

[26] Th. M. Rassias and K. Shibata, Variational problem of some quadratic functionals in complex analysis, J. Math. Anal. Appl. 228 (1998), 234-253.

[27] F. Skof, Local properties and approximation of operators, Rend. Sem. Mat. Fis. Milano 53 (1983), 113-129.

[28] T. Trif, On the stability of a functional equation deriving from an inequality of Popoviciu for convex functions, J. Math. Anal. Appl. 272 (2002), no. 2, 604-616.

[29] S. M. Ulam, Problems in Modern Mathematics, Science Editions, Wiley, New York, 1964. 
ChoOnkil Park

Department of Mathematics

Research Institute for Natural Sciences

HANYANG UNIVERSITY

SEOUl 133-791, KoreA

E-mail address: baak@hanyang.ac.kr

M. Eshaghi Gordu

Department of Mathematics

Semnan University

P. O. Box 35195-363, SEmnan, Iran

E-mail address: madjid.eshaghi@gmail.com

H. KHODAEI

Department of Mathematics

Semnan University

P. O. Box 35195-363, SEmnan, Iran

E-mail address: khodaei.hamid.math@gmail.com 